\title{
Lerngelegenheiten mit kaufmännischem Kontext im Mathematikunterricht der allgemeinbildenden Schule - Eine Schulbuch- und Aufgabenanalyse
}

\author{
Robert von Hering $(\mathbb{D} \cdot$ Halima Zingelmann • Aiso Heinze • \\ Anke Lindmeier
}

Online publiziert: 29. Januar 2020

(C) Der/die Autor(en) 2020

Zusammenfassung Der Erwerb mathematischer Kompetenz im allgemeinbildenden Unterricht soll unter anderem der Berufsvorbereitung dienen. Für das Fach Mathematik sind kaufmännische Ausbildungsberufe mit ihren hohen Ausbildungszahlen und mathematischen Anforderungen eine wichtige Referenz. Werden in der Schule berufsfeldbezogene mathematische Kompetenzen erworben, so können diese theoretisch Passungsprobleme am Übergang Schule - Beruf abmildern. Es stellt sich daher die Frage, inwiefern im allgemeinbildenden Mathematikunterricht vorbereitende Lerngelegenheiten für den kaufmännischen Bereich (exemplarisch für Industriekaufleute) vorkommen. In der Studie wurden als Annährung an den Unterricht Schulbücher der Sekundarstufe im Hinblick auf Aufgaben mit (industrie-) kaufmännischem Kontext untersucht. Die Klassifikation der Kontexte von 18 allgemeinbildenden Mathematikschulbüchern aus 4 Reihen zeigt, dass zwar 6\% der Aufgaben ein Potential zur Vorbereitung auf kaufmännische Anforderungen enthalten, die Aufgaben weisen aber größtenteils einen niedrigen Grad an Berufsbezogenheit auf. Die Studie kann zum besseren Verständnis der derzeitigen Ausgestaltung von Berufsvorbereitung im allgemeinbilden Mathematikunterricht beitragen.

Schlüsselwörter Berufsvorbereitung · Übergang Schule - Beruf · MathematikSchulbuch · Mathematische Kompetenzen

R. von Hering $(\bowtie) \cdot$ Prof. Dr. A. Heinze $\cdot$ Prof. Dr. A. Lindmeier Didaktik der Mathematik, IPN - Leibniz-Institut für die Pädagogik der Naturwissenschaften und Mathematik, Olshausenstraße 62, 24118 Kiel, Deutschland

E-Mail: hering@leibniz-ipn.de

Prof. Dr. A. Heinze

E-Mail: heinze@leibniz-ipn.de

Prof. Dr. A. Lindmeier

E-Mail: lindmeier@leibniz-ipn.de 


\title{
Learning opportunities with commercial context in secondary mathematics instruction-An analysis of textbooks and tasks
}

\begin{abstract}
The acquisition of mathematical competence in general education should, among other things, serve as vocational preparation. Commercial vocations hold a great amount of mathematical demands, involve a great number of trainees, so they should be an important reference for the subject of mathematics. If vocationrelated mathematical competencies were already acquired at school, they could probably reduce difficulties at the transition from school to work. The question, therefore, arises as to what extent preparatory learning opportunities for the commercial sector (shown by the example of industrial clerks) are part of general mathematics education. In the present study, secondary school textbooks were examined as a reference to the teaching praxis and the mathematical tasks used in class. More precisely, textbook tasks were analyzed with regard to their commercial vocational context. The classification of the contexts of 18 general education mathematics textbooks from 4 series shows that $6 \%$ of the tasks have the potential for preparing commercial contents. However, the tasks mostly have a low degree of occupation-relatedness. The study may help to better understand the current design of vocational preparation in general mathematics education.
\end{abstract}

Keywords Mathematical competencies - Mathematics textbook - Transition from school to work $\cdot$ Vocational preparation

\section{Einleitung und Motivation}

Die Lebensvorbereitung, also auch die Berufsvorbereitung, ist nach Heymann (1996) ein Ziel des allgemeinbildenden Mathematikunterrichts. Durch die Bildungsstandards im Fach Mathematik für den Mittleren Schulabschluss sowie für die Allgemeine Hochschulreife ist eine fachliche Berufsvorbereitung als Teilaspekt der mathematical literacy (OECD 2003) entsprechend als Bildungsziel verankert (KMK 2003).

Beim Übergang von der allgemeinbildenden Schule in die berufliche Erstausbildung erweisen sich nicht nur aus bildungstheoretischer, sondern auch aus empirischer Sicht mathematische Kompetenzen als eine zentrale Größe, was den Stellenwert der Berufsvorbereitung durch die allgemeinbildende Schule unterstreicht (Lehmann und Seeber 2007). Gleichzeitig kann wegen der verschiedenen Bildungskonzeptionen nicht von einem kontinuierlichen kumulativen Kompetenzaufbau beim Übergang von der Schule in die Berufsbildung ausgegangen werden (Heinze und Grüßing 2009). Es ist bisher insbesondere unklar, welche Rolle berufsvorbereitende Lernangebote im allgemeinbildenden Mathematikunterricht tatsächlich spielen.

Soll diese Lücke geschlossen werden, so tritt das Problem auf, dass in verschiedenen Berufsfeldern sehr unterschiedliche mathematische Anforderungen auftreten. Es ist daher naheliegend, in einem ersten Zugriff typische Berufsfelder mit erkennbarem mathematischem Bezug in den Blick zu nehmen. Exemplarisch können dabei die kaufmännischen Ausbildungsberufe aus dem in Deutschland mit Abstand 
größten Ausbildungssektor Industrie \& Handel herangezogen werden, da hier viele Ausbildungsplätze angeboten werden und die Ausbildungsinhalte einen hohen mathematischen Anteil aufweisen. In diesem Beitrag wird daher der Frage nachgegangen, welche berufsvorbereitenden Lerngelegenheiten für den kaufmännischen Bereich es im allgemeinbildenden Mathematikunterricht der Sekundarstufe gibt.

Als Indikator für solche vorbereitenden Lernangebote für kaufmännische Berufe wird die Implementation von kaufmännischen Kontexten im Mathematikunterricht betrachtet. In dieser Studie wurden dabei Mathematikschulbücher bzw. die darin enthaltenen Aufgaben als eine Näherung für die Implementation curricularer Vorgaben im Fachunterricht genutzt.

\section{Theoretischer Hintergrund}

\subsection{Die kaufmännische Ausbildung am Beispiel der Industriekaufleute}

Die berufliche Ausbildung wird in Deutschland meist im dualen Ausbildungssystem (Betrieb und Berufsschule) durchgeführt und genießt weltweit ein hohes Ansehen (Jongebloed 2011). In den letzten Jahren lässt sich dabei beobachten, dass die Anforderungen an Auszubildende im Vergleich zu den von den Ausbildungsbetrieben angegebenen Qualifikationen anspruchsvoller sind (Münk 2013). Aufgrund des dadurch entstehenden Passungsproblems erweist sich die Eingliederung in die neue Ausbildungsinstitution für eine substanzielle Anzahl an Jugendlichen als problematisch (Münk 2013).

Der Wirtschaftssektor Industrie \& Handel stellt in Deutschland mit Abstand die meisten Ausbildungsplätze. Im Jahr 2017 fielen von allen neu begonnenen Ausbildungen $58 \%$ in diesen Sektor, gefolgt vom Handwerk mit $28 \%$ der Ausbildungsverträge (BIBB 2018). Wie in den Jahren zuvor befindet sich der Ausbildungsberuf Industriekaufmann/-frau neben drei weiteren kaufmännischen Berufen unter den Top 5 der neu abgeschlossenen Ausbildungsverträge (BIBB 2018). Damit kann der kaufmännische Ausbildungsbereich im Allgemeinen, der Beruf Industriekaufmann/ -frau im Speziellen als wichtiges Referenzkriterium für die Berufsvorbereitung im allgemeinbildenden Mathematikunterricht angesehen werden. Im Folgenden sollen daher am Beispiel der Industriekaufleute Anforderungen der kaufmännischen Ausbildung dargestellt werden.

Die Ausbildung der Industriekaufleute dauert 3 Jahre, findet dual und gleichberechtigt in Betrieb und beruflicher Schule statt und ist durch den Rahmenlehrplan der KMK und die Ausbildungsordnungen des Bundes für die betriebliche Ausbildung geregelt (KMK 2002; BMBF 2002). Der Anteil der Auszubildenden mit Studienberechtigung ist bei den Industriekaufleuten überdurchschnittlich hoch. Während der Anteil der Auszubildenden mit mindestens Fachhochschulreife über alle Ausbildungsberufe hinweg in Deutschland bei $29 \%$ liegt, sind es in den kaufmännischen Ausbildungsberufen $35 \%$ und bei den Industriekaufleuten sogar 70\% (BIBB 2018). In einer in drei Bundesländern durchgeführten Studie mit 650 angehenden Industriekaufleuten belief sich die Abiturientenquote auf $53 \%$ (Volodina et al. 2015). Es 
kann also angenommen werden, dass die Anforderungen des Ausbildungsberufs der Industriekaufleute im Vergleich zu anderen Berufen erhöht sind.

Darüber hinaus zeichnet sich die Ausbildung der Industriekaufleute durch einen hohen Mathematikgehalt aus. Der Rahmenlehrplan der KMK (2002) unterteilt die Ausbildungsinhalte in 12 Lernfelder, von denen fünf einen starken mathematischen Bezug aufweisen. Die relevanten Inhalte lassen sich in die vier Kategorien Kostenrechnung, Leistungsrechnung, Abschreibung sowie Finanzierung und Investition unterteilen (vgl. KMK 2002). Aus mathematisch-fachlicher Sicht entsprechen die Anforderungen vornehmlich Anwendungen von Dreisatz und Prozentrechnung, wobei das Niveau des allgemeinbildenden Mathematikunterrichts der Sekundarstufe I aus fachlicher Sicht nicht überschritten wird. Im Gegensatz zum Schulunterricht ist in der Ausbildung aber die Bewältigung von beruflichen Anforderungen unter - teils impliziter - Nutzung von mathematischen Mitteln zentral. Das heißt, Mathematik wird immer innerhalb von beruflichen Kontexten angewendet und nicht fachliche (z. B. linearer Zusammenhang), sondern beruflich geprägte Strukturierungen (z.B. Abschreibung, Stückkosten) sind leitend. Entsprechend kommt der kaufmännischen Fachsprache im Vergleich zur Schulmathematik eine hohe Bedeutung zu.

Anhand des Ausbildungsberufs Industriekaufmann/-frau wird deutlich, dass kaufmännische Berufe eine große Nähe zum Unterrichtsfach Mathematik aufweisen. Wenn also berufsvorbereitende Aspekte im allgemeinbildenden Mathematikunterricht vorkommen, dann wäre es naheliegend, dass diese Berufe, die eine hohe Auszubildendenanzahl aufweisen und gleichzeitig als mathematik-affin zu charakterisieren sind, eine erkennbare Rolle spielen.

\subsection{Kompetenzbegriffe und -modelle am Übergang Schule - Berufsausbildung}

Beim Übergang von der allgemeinbildenden Schule in die berufliche Erstausbildung ändern sich die Anforderungen für die Lernenden auf vielfältige Weise. Insbesondere betrifft dies auch die Art der zu erwerbenden Kompetenzen, da Schule und Berufsbildung grundsätzlich unterschiedliche Zielsetzungen verfolgen. Dies hat Auswirkungen auf den Verlauf des individuellen Kompetenzerwerbs, sodass nicht zwangsläufig von kontinuierlichem, kumulativem Kompetenzaufbau am Übergang Schule - Beruf ausgegangen werden kann (Heinze und Grüßing 2009; Neumann et al. 2013).

Im beruflichen Kontext spielt der Begriff der Handlungskompetenz eine zentrale Rolle (Nickolaus 2011). Mit diesem Begriff wird Kompetenz als die Fähigkeit, komplexe berufliche Anforderungssituationen bewältigen zu können, verstanden. Kompetenz ist damit eng mit beruflicher Performanz verbunden. Das Ziel der beruflichen Handlungskompetenz umfasst die Entwicklung einer beruflichen Mündigkeit, sodass das Referenzkriterium für Kompetenzerwerb also die beruflichen Anforderungen sind (Nickolaus und Seeber 2013).

Im Gegensatz dazu beziehen sich Kompetenzen im allgemeinbildenden Bereich auf ,kontextspezifische kognitive Leistungsdispositionen, die sich funktional auf Situationen und Anforderungen in bestimmten Domänen beziehen" (Klieme und Leutner 2006, S. 879). Statt des beruflichen Referenzrahmens - und damit einem Performanzbezug - ist der hier verwendete Kompetenzbegriff ganz im Sinne des 
allgemeinbildenden Auftrags der Schule auf den Erwerb kognitiver Grundlagen bezogen, die in fachlicher Strukturierung, beispielsweise als mathematische Kompetenzen, erworben werden sollen. Sie tragen damit zur Bildung im Sinne einer Grundbildung bei, die insbesondere auf eine breite Anwendbarkeit der erworbenen Kompetenzen abzielt und gleichzeitig den Erwerb der zugrundeliegenden Wissensstrukturen betont, was sich beispielsweise in den Bildungsstandards Mathematik (KMK 2003) abbildet.

Kontrastiert man diese beiden Kompetenzverständnisse, so ergibt sich auf Basis der theoretischen Grundlagen ein dreiteiliges Strukturmodell der Kompetenzen, die am Übergang Schule - Beruf aus fachlicher Sicht relevant erscheinen (Neumann et al. 2013; vgl. auch Winther 2010). Zum einen werden - wie eben skizziert - allgemeine mathematische Kompetenzen als Ziel der allgemeinbildenden Schule von den beruflichen Kompetenzen abgegrenzt. Zum Überbrücken des theoretischen Bruchs bieten sich berufsfeldbezogene mathematische Kompetenzen (Neumann et al. 2013) an. Diese ergeben sich im Überschneidungsbereich von allgemeinbildenden und beruflichen Kompetenzen. Sie kommen zum Tragen, wenn im Beruf mathematikhaltige Anforderungssituationen vorkommen, die unter der Nutzung allgemeinbildender mathematischer Kompetenzen bewältigt werden können.

Mit Blick auf den Übergang Schule - Ausbildung verfügen Auszubildende (im Regelfall) bereits zu Beginn der Ausbildung über berufsfeldbezogene mathematische Kompetenzen, da der allgemeinbildende Unterrichts auf die breite, also auch berufsbezogene Anwendbarkeit der mathematischen Kompetenzen abzielt. Zudem sollten berufsfeldbezogene mathematische Kompetenzen den Erwerb beruflicher Kompetenzen erleichtern, da sie bereits einfache kaufmännische Anforderungssituationen abbilden und somit ein Anknüpfungspunkt für komplexere berufliche Anforderungen sein können. Sie haben also theoretisch das Potenzial, die Diskontinuität im individuellen Kompetenzerwerb am Übergang Schule - Beruf abzumildern. Erste Untersuchungen deuten auf eine empirische Trennbarkeit der allgemeinen mathematischen und berufsfeldbezogenen Kompetenzen bei Auszubildenden zu Beginn der Ausbildung hin (Siebert und Heinze 2016). Auch qualitativ konnten diese Ergebnisse in einer Studie (Parallelaufgaben mit und ohne beruflichen Kontext und anschließenden Interviews) mit Industriekaufleuten in der Ausbildung bestätigt werden (von Hering et al. 2018).

Zusammenfassend erscheint die Betrachtung eines solchen berufsfeldbezogenen mathematischen Kompetenzbereichs aus theoretischer Sicht zur Beschreibung der (Nicht-)Anschlussfähigkeit von Bildungsprozessen am Übergang Schule - Berufsschule geeignet. Es ist bisher aber unklar, inwiefern es aus forschungspraktischer Sicht nützlich ist, innerhalb der allgemeinbildenden mathematischen Kompetenzen feinkörnig einen Teilbereich berufsfeldbezogener mathematischer Kompetenzen abzugrenzen. Da die Unterscheidung sich wie dargelegt aus den zu bewältigenden Anforderungen, also dem Kontext für die Anwendung von Mathematik, speist, soll im Folgenden vertieft werden, welche Bedeutung Kontexte beim Erwerb mathematischer Kompetenzen haben. 


\subsection{Bedeutung des Kontextes beim Erwerb mathematischer Kompetenz}

In ihrer Definition von Mathematical Literacy formuliert die OECD (2003) mathematische Kompetenz als die Fähigkeit, in vielfältigen Kontexten Mathematik zu nutzen. Es stellt sich also die Frage, wie dies durch den allgemeinbildenden Unterricht erreicht werden kann. Aus der kognitionspsychologischen Forschung ist bekannt, dass die Übertragung von Fähigkeiten zwischen verschiedenen Kontexten schwierig ist (Transferproblem, zsf. Perkins und Salomon 1992), vor allem wenn Kontexte wenig ähnlich sind (sog. ferner Transfer).

Dies lenkt die Aufmerksamkeit auf die im Unterricht genutzten Kontexte. Das Transferproblem und zugehörige Erkläransätze legen nahe, die Kontexte als integrale Bestandteile der Lerngelegenheit und nicht etwa im Gegensatz dazu als austauschbare Gestaltungselemente von Lerngelegenheiten zu verstehen. Die Cognitive flexibility theory (Spiro et al. 1988) beispielsweise führt erfolgreiches Problemlösen auf die Qualität der erworbenen Kompetenzen zurück. Werden bereits beim Erwerb verschiedene Anwendungssituationen eingesetzt, so führe dies zu höherer kognitiver Flexibilität, da es keine oder eine einseitige Verknüpfung der Kompetenzen mit Anwendungen verhindert. Noch stärkere Annahmen setzt beispielsweise die Situated cognition theory (Brown et al. 1989). Hier geht man davon aus, dass Kompetenzen in den Erwerbskontexten situiert sind und somit Anwendungs- nicht von Erwerbskontexten trennbar sind. Für den allgemeinbildenden Mathematikunterricht würde diese Annahme ebenfalls bedeuten, dass vielfältige Anforderungssituationen bereits beim Erwerb der mathematischen Kompetenzen genutzt werden sollten. $\mathrm{Zu}$ sammenfassend und im Anschluss an die Forschungslage wird für den Erwerb von Mathematical Literacy im Mathematikunterricht empfohlen, insbesondere auch Aufgaben zu nutzen, die - kongruent zur Zielsetzung - die Anwendung von Mathematik in realistischen, auch berufsbezogenen Kontexten erfordern (OECD 2003).

In der Mathematikdidaktik werden solche Kompetenzen spezifischer als mathematische Modellierungskompetenzen beschrieben. Darunter versteht man die Fähigkeiten, Aufgaben mit Situationsbezug unter Einsatz mathematischer Modelle zu lösen (Klieme et al. 2001; KMK 2003). Dazu muss u. a. die Situation in ein mathematisches Modell übersetzt, innermathematisch bearbeitet und die mathematische Lösung vor dem Hintergrund der Situation interpretiert werden, sodass Teilkompetenzen beschrieben werden können (Blum und Leiß 2005).

Niss und Højgaard (2011) arbeiten Qualitätsdimensionen für mathematische Modellierungskompetenzen heraus. Dabei beschreibt Radius of action die Reichweite, also auf welche Bandbreite an Kontexten und Situationen Modellierungskompetenzen angewendet werden können, was die Kernfrage des Transferproblems aufgreift. Es wird angenommen, dass der Erwerb funktionaler Modellierungskompetenzen davon abhängt, ob Lerngelegenheiten in relevanten Kontexten und Situationen vorkommen, der angezielte Radius of action also bereits beim Erwerb berücksichtigt wird. Dabei können berufliche oder private Kontexte genutzt werden, die dann als sinnstiftende Probleme zum Erwerb von Handlungsschemata im Sinne des Prinzips der Problemorientierung beitragen (Euler und Hahn 2014). Betrachtet man feinkörniger die Art der im Mathematikunterricht genutzten Realitätsbezüge, so lassen sich verschiedene Grade an Authentizität erkennen. Die Bandbreite reicht von soge- 
nannten eingekleideten Aufgaben, die sich in erfundenen und meist austauschbaren Realsituationen abspielen, bis hin zu authentischen Aufgaben mit Lebensweltbezug, wobei den verschiedenen Typen gleichermaßen spezifische Funktionen und Berechtigungen im Unterricht zugeschrieben werden können (Leuders und Leiß 2006). Leuders und Leiß (2006) empfehlen entsprechend für Unterricht und Schulbuch eine Ausgewogenheit, insbesondere können Realitätsbezüge nicht als alleiniges Qualitätskriterium verstanden werden. Authentische Realitätsbezüge gelten jedoch als besonders geeignetes Mittel, um die Rolle und Bedeutsamkeit der Mathematik in der Welt zu verstehen.

Übereinstimmend wird also davon ausgegangen, dass die beim Erwerb allgemeiner mathematischer Kompetenzen genutzten Kontexte beeinflussen, inwiefern die Kompetenzen in anderen Zusammenhängen genutzt werden können. Demgegenüber steht eine lückenhafte Forschungslage in Bezug auf die tatsächlich genutzten Kontexte im Mathematikunterricht der allgemeinbildenden Schule. Die wenigen Forschungserkenntnisse beziehen sich auf die Unterscheidung von außer- und innermathematischen Kontexten (in Deutschland ca. 11\% der Aufgaben mit außerund $2 \%$ mit innermathematischem Kontext in TIMSS-Video 1999), differenzieren jedoch bisher nicht bezüglich unterschiedlicher außermathematischer Kontexte (z. B. Neubrand 2002; Jordan et al. 2006). Es liegen bisher also keine Anhaltspunkte vor, inwiefern berufliche Kontexte im allgemeinbildenden Mathematikunterricht auftreten.

\subsection{Das Schulbuch als potentiell implementiertes Curriculum}

In der hier berichteten Studie sollen Erkenntnisse über die potentiell berufsvorbereitenden Lerngelegenheiten im allgemeinbildenden Mathematikunterricht der Sekundarstufe erlangt werden. Dazu wurden in einem ersten Zugriff die Lerngelegenheiten mit beruflichem Bezug aus Mathematikschulbüchern untersucht. Diese zentrale methodische Entscheidung soll im Folgenden begründet werden. Im Rahmen der TIMSS-Studien wurde ein Modell verschiedener Curriculumsebenen entwickelt, das die Vermittlungsprozesse zwischen dem intendierten Curriculum (z. B. Bildungsstandards, Lehrpläne) und dem implementierten Curriculum (den Lehr-Lernaktivitäten im Unterricht, die das Lernangebot darstellen) beschreibt (Valverde et al. 2002). Unterrichtsmaterialien, also insbesondere auch Schulbücher, werden dort als potentiell implementiertes Curriculum bezeichnet und in der Ebene zwischen intendiertem und implementiertem Curriculum als zentrale Vermittlungsressource verortet. Soll das Schulbuch auf dieser Basis als Indikator für unterrichtliche Merkmale genutzt werden, so muss abgeschätzt werden, inwiefern der Schluss von Merkmalen des Schulbuchs auf das tatsächlich implementierte Curriculum gerechtfertigt ist.

Studien zur Schulbuchnutzung haben ergeben, dass das Mathematik-Schulbuch für $86 \%$ der Mathematiklehrkräfte an deutschen Grundschulen die Hauptquelle für die Unterrichtsvorbereitung ist (OECD-Durchschnitt: 75\%, Mullis et al. 2012). Eine ähnliche Untersuchung für weiterführende Schulen gibt es für Deutschland leider nicht. Allerdings zeigte sich für andere Nationen, dass die Werte für Grundschulen und weiterführende Schulen eine hohe Konsistenz aufweisen (Mullis et al. 2012). Entsprechend kann angenommen werden, dass auch hier das Mathematikschulbuch 
die zentrale Ressource für die Unterrichtsvorbereitung darstellt. Dazu zeigen Studien einen positiven Zusammenhang zwischen dem Umfang eines Themas in Schulbüchern und entsprechenden Merkmalen des Unterrichts bzw. der Leitungsentwicklung (Schmidt et al. 2001; van den Ham und Heinze 2018). Somit kann das Schulbuch im Sinne des potentiell implementierten Curriculums als Annäherung für Merkmale des Mathematikunterrichts genutzt werden. Dabei wird die Kultur vom Mathematikunterricht stark durch die Verwendung von Aufgaben geprägt, die auch als strukturgebende Elemente der Mathematikschulbücher fungieren (Neubrand 2002). Sollen also Anhaltspunkte gewonnen werden, inwiefern potentielle berufsfeldbezogene Lerngelegenheiten im Unterricht auftreten, so ist es sinnvoll, zur Operationalisierung die Aufgaben aus den Schulbüchern und die darin vorkommenden Kontexte heranzuziehen.

Andere Untersuchungen auf Basis von Schulbuchanalysen nehmen bislang statt fachlich-inhaltlicher Aspekte vornehmlich Strukturmerkmale auf Aufgaben-, Kapitel- oder Buchebene in den Blick (z. B. Rezat 2009). Insbesondere Analysen, die Kontexte von Aufgaben im Allgemeinen bzw. Gelegenheiten zur Berufsorientierung für den Übergang von der allgemeinbildenden Schule in die berufliche Erstausbildung im Speziellen adressieren, stehen bislang aus.

\subsection{Forschungsfragen}

Berufsfeldbezogene mathematische Kompetenzen erscheinen theoretisch als Mittler zwischen den unterschiedlichen Anforderungen in der allgemeinbildenden Schule und im Beruf und haben so das Potential, die Diskontinuität im individuellen Kompetenzerwerb zu ebnen, sodass die schulischen mathematischen Kompetenzen funktional in berufliche Kompetenzen integriert werden können (Neumann et al. 2013). Bisher ist aber unklar, inwiefern sich Lerngelegenheiten für berufsfeldbezogene Kompetenzen bereits im allgemeinbildenden Mathematikunterricht ergeben.

Der kaufmännische Berufszweig und konkret der Ausbildungsberuf Industriekaufmann/-frau stehen ob ihrer Nähe zum Fach Mathematik im Fokus dieser Studie. Das Schulbuch kann im Sinne des potentiell implementierten Curriculums stellvertretend für den Mathematikunterricht betrachtet werden. Die übergeordnete Frage nach Lerngelegenheiten für berufsfeldbezogene mathematische Kompetenzen im allgemeinbildenden Mathematikunterricht führt zu folgenden Forschungsfragen, die auf eine Deskription der vorliegenden Praxis zielen:

1. Wie hoch ist der Anteil der Aufgaben in Mathematikschulbüchern, die in einem kaufmännischen Kontext stehen?

2. Wie hoch ist der Anteil dieser Aufgaben mit kaufmännischem Kontext, die eine berufsbezogene Perspektive bzw. eine industriekaufmännische Perspektive einnehmen?

3. Gibt es hinsichtlich der Ergebnisse der Forschungsfragen 1 und 2 Unterschiede bezüglich der Klassenstufen oder Schulbuchreihen?

Die erste Forschungsfrage zielt auf die Identifikation solcher Aufgaben und Lerngelegenheiten, die einen kaufmännischen Bezug aufweisen. Dabei kann es sich, im Sinne des Prinzips der Problemorientierung (Euler und Hahn 2014) bei den kaufmän- 
nischen Kontexten sowohl um eine private wie auch um eine berufliche Perspektive handeln. Die zweite Frage beschäftigt sich mit der Teilmenge der Aufgaben und Lerngelegenheiten, die spezifisch die Perspektive der beruflichen Anforderungen einnehmen. Diese können im engeren Sinne als authentische berufsvorbereitende Lerngelegenheiten eingestuft werden. Dabei wird noch einmal in industriekaufmännische Kontexte und andere berufsbezogene kaufmännische Kontexte differenziert.

Die dritte Forschungsfrage zu Unterschieden bezüglich der Klassenstufen und Schulbuchreihen kann über die zeitliche Passung von berufsorientierenden Angeboten sowie über die auftretende Varianz Auskunft geben. Dabei interessiert, ob in höheren Klassenstufen aufgrund der zeitlichen Nähe zum Übergang in die Berufsausbildung vermehrt berufsfeldbezogene mathematische Kompetenzen angesprochen werden. Ebenso wäre es plausibel anzunehmen, dass Schulbücher, die sich primär an Bildungsgänge für den Mittleren Schulabschluss wenden, mehr berufsfeldbezogene mathematische Lerngelegenheiten aufweisen als solche, die sich primär auf Bildungsgänge mit dem Ziel des Erwerbs der Hochschulzugangsberechtigung wenden.

\section{Methodisches Vorgehen}

\subsection{Auswahl der Schulbücher und Festlegung der Analyseeinheiten}

Für die Identifikation und Analyse von Lerngelegenheiten mit kaufmännischem Kontext im Mathematikunterricht haben wir Schulbücher im Sinne des potentiell implementierten Curriculums als Annäherung an das implementierte Curriculum genutzt. Die Auswahl der untersuchten Schulbücher orientierte sich an der üblichen schulischen Vorbildung der kaufmännischen Auszubildenden. Beispielsweise verfügen fast alle angehenden Industriekaufleute mindestens über den Mittleren Schulabschluss, ein großer Anteil besitzt aber auch eine Hochschulzugangsberechtigung (BIBB 2018). Daher haben wir für unsere Analyse Mathematikschulbücher für Gymnasien und weitere Schulformen, die zum Mittleren Schulabschluss führen, herangezogen. Dabei wurden nur Bücher der Sekundarstufe I berücksichtigt, da die Berufsorientierung für Ausbildungsberufe typischerweise zum Mittleren Schulabschluss erfolgt und auch die im Beruf genutzten mathematischen Inhalte in diesen Klassen verortet sind.

Der betrachtete Korpus umfasst insgesamt 18 Mathematikschulbücher aus vier verschiedenen Reihen von drei Schulbuchverlagen (Baum et al. 2006-2009; Böer et al. 2008, 2009, 2013, 2014; Cukrowicz et al. 2005-2009; Lergenmüller \& Schmidt 2005-2009), die sich jeweils curricular an den Anforderungen mehrerer Bundesländer orientieren. Dabei richten sich alle Reihen auch an eine gymnasiale G9-Struktur. Die Reihe „mathelive“ zielt im Schwerpunkt auf den Mathematikunterricht in Haupt-, Real-, Gesamt- und Gemeinschaftsschule ab. Es wurden die Schulbücher für die Klassenstufe 7 bis 10 betrachtet, bei zwei Reihen zusätzlich die Schulbücher der Klassenstufe 6. So kann ein umfangreicher Überblick über die potentiellen Lerngelegenheiten der Sekundarstufe I an verschiedenen Schulformen und ohne Einschränkung auf ein Bundesland gegeben werden. 
Die Analyse sollte sämtliche Lerngelegenheiten erfassen, wobei unter Lerngelegenheit nicht nur Aufgaben im engeren Sinne (meist durch eine Nummer gekennzeichnet), sondern auch Elemente wie Informationstexte, Merksätze und Beispiele verstanden wurden. Diese werden im Folgenden alle unter den Terminus Aufgabe gefasst, da es sich beim Großteil der angebotenen Lerngelegenheiten um klassische Aufgaben handelt, was die den Mathematikunterricht prägende Aufgabenkultur widerspiegelt (Neubrand 2002). Aufgaben mit mehreren Teilen betrachten wir dabei als eine Einheit, da der Kontext in der Regel identisch ist. Die so verstandenen Aufgaben bilden die Analyseeinheiten dieser Studie. Es ergeben sich auf diese Weise in den 18 Schulbüchern eine Grundgesamtheit von etwa 20.000 Aufgaben ${ }^{1}$, von denen etwa $33 \%$ eine Kontextualisierung aufweisen und somit für eine feinkörnigere Analyse in Bezug auf den eventuellen Berufsbezug von Interesse sind.

\subsection{Kategoriensystem für die Klassifikation nach Inhalten und Berufsbezug}

Die Aufgaben wurden in einem zweistufigen System kategorisiert: Zunächst wurde in einem Identifikations-Schritt für jede der 20.000 Aufgaben überprüft, ob ein kaufmännischer Kontext vorliegt. Die identifizierten Aufgaben wurden in einem anschließenden Klassifizierungs-Schritt bezüglich verschiedener Kategorien einsortiert.

Indikatoren für die Identifikation eines kaufmännischen Kontextes im ersten Schritt sind Begriffe, die auf einen kaufmännischen Betrieb hinweisen (z. B. ein Hotel, allgemein ein Unternehmen oder eine Firma, eine Werkstatt, ein Modegeschäft etc.), oder Begriffe und Formulierungen, bei denen es um Einnahmen, Ausgaben oder Finanzen im Allgemeinen geht (z. B. Absatz, Zinsen, Gewinne).

Im zweiten Schritt (Klassifizierung) erfolgt eine leitfragen- und beispielgesteuerte Zuweisung zu Kategorien. Dabei werden die zwei Bereiche Mathematischer Inhalt und Grad der Berufsbezogenheit berücksichtigt. Die Klassifizierungskriterien für den mathematischen Inhalt wurden aus den Rahmenlehrplänen für Industriekaufleute in der Ausbildung (KMK 2002) gewonnen. Die dort genannten und von uns gebündelten vier mathematischen Inhaltskategorien Kostenrechnung, Leistungsrechnung, Abschreibung sowie Finanzierung/Investition bilden die mathematisch-inhaltlichen Kategorien. Tab. 1 zeigt exemplarisch Leitfragen und Ankerbeispiele für die inhaltliche Kategorie Finanzierung/Investition. So gelten beispielsweise Übungsaufgaben zur Automatisierung von Prozeduren der Prozentrechnung ohne Kontexte, selbst wenn mit Geldbeträgen gerechnet wird (z.B. „Berechne $35 \%$ von $78 €$.“), nicht als kaufmännische Aufgaben im Sinne einer der vier Inhaltskategorien.

Für die Klassifikation nach dem Grad der Berufsbezogenheit wurden drei Kategorien genutzt, die die kontextuelle Nähe zum Beruf der Industriekaufleute angeben. Die Kategorie industriekaufmännischer Kontext ist dabei spezifisch berufsbezogen. Es handelt sich um Aufgaben, deren Kontexte das Tätigkeitsfeld von Industriekaufleuten spiegeln. Eine Aufgabe wird dieser Kategorie zugeordnet, wenn z.B. der

\footnotetext{
${ }^{1}$ Im Rahmen der Erstkodierung wurde die Aufgabenzahl nicht erfasst. Die Zweitkodierung von vier Schulbüchern verschiedener Klassenstufen und Schulbuchreihen erbrachte insgesamt 4351 Aufgaben, sodass diese Studie auf etwa 20.000 Aufgaben basiert.
} 
Tab. 1 Kriterien zur Klassifizierung des Mathematischen Inhalts, hier am Beispiel der Kategorie Finanzierung/Investition

Kategorie Finanzierung/Investition

\begin{tabular}{ll}
\hline Allgemeine Leitfragen & $\begin{array}{l}\text { Wie hoch sind die Zinsen für einen Kredit? } \\
\text { Auf welche Weise soll eine Rechnung beglichen werden? }\end{array}$ \\
$\begin{array}{l}\text { Eine Lerngelegenheit wird } \\
\text { Finanzierung/Investition }\end{array}$ & $\begin{array}{l}\text {... es um Guthaben auf Konten geht. } \\
\text { zugeordnet, wenn ... }\end{array}$ \\
$\begin{array}{l}\text {... es um Aufgaben zu Kapital und Zinsen bei der Bank geht. } \\
\text {... es bspw. um Ratenzahlung oder Finanzierungsmöglichkeiten geht. } \\
\text { Fine Lerngelegenheit wird } \\
\text { Finanzierung/Investition } \\
\text { nicht zugeordnet, wenn ... }\end{array}$ & ... Versicherungen das zentrale Thema sind. \\
\end{tabular}

Handel, Kauf oder Verkauf von industriell hergestellten Produkten thematisiert wird oder eine Frage aus der Perspektive eines Industrieunternehmens beantwortet werden soll. Als Indikator wurde auch die Nutzung einschlägiger Fachtermini gewertet. Einen mittleren Grad an Berufsbezogenheit zum Industriekaufmannswesen stellt die Kategorie andere berufsbezogene kaufmännische Kontexte dar. Eine Aufgabe wird dieser Kategorie zugeordnet, wenn andere kaufmännische Berufe oder Unternehmen genannt werden oder wenn mathematische Prinzipien oder Rechenwege angewendet werden müssen, die den industriekaufmännischen entsprechen, aber die Aufgabe nicht im engeren Sinne in einem industriekaufmännischen Kontext situiert ist (vgl. Erläuterung zu Abb. 2). Diese Kategorie umfasst also kaufmännische Kontexte mit Berufsbezug aber ohne industriekaufmännischer Spezifizierung. Schließlich wurde eine dritte Kategorie für Aufgaben mit niedrigem Berufsbezug genutzt, die Klasse der privaten/nicht eindeutigen kaufmännischen Kontexte. Diese wurde angewendet, wenn ein kaufmännischer oder allgemein wirtschaftlicher Themenbereich mit kaufmännischem Zusammenhang aus der Perspektive einer Privatperson oder aus einer neutralen Perspektive betrachtet wird (vgl. Erläuterung zu Abb. 3). Als Indikator diente, dass die Schülerin oder der Schüler durch die Aufgabe direkt mit einem „Du“ angesprochen wird, Signalbegriffe aus dem privat konnotierten Bereich (z.B. Sparbuch, Handytarif, Busfahrkarte, Taschengeld) vorkommen oder ein allgemein wirtschaftlicher Kontext ohne Berufsbezug vorkommt. Aufgaben aus dieser dritten Kategorie weisen somit keinen oder keinen einschlägigen Berufsbezug auf. Die Kategorisierung wird im Folgenden an Beispielen illustriert.

Abb. 1 zeigt eine Schulbuchaufgabe, die inhaltlich in den Bereich der Kostenrechnung fällt. Durch die Betrachtung der kaufmännischen Situation aus der Sicht eines Industrieunternehmens, durch den Kontext (Verkauf von industriell hergestellten Produkten) sowie durch die kaufmännische Fachsprache (vgl. Termini am Aufgabenrand) wird sie der Kategorie industriekaufmännischer Kontext zugewiesen (hoher Grad an Berufsbezogenheit).

Auch Abb. 2 zeigt eine Aufgabe aus der inhaltlichen Kategorie Kostenrechnung. Der Kontext ist ebenfalls dem kaufmännischen Bereich zuzuordnen (Gewinnberechnung). Im Gegensatz zur Aufgabe in Abb. 1 wird hierbei jedoch die Perspektive eines Einzelhandelskaufmanns angenommen, auch wenn ,Zeitungsverkäufer“ nicht zwangsläufig eine Ausbildung für Einzelhandelskaufleute absolvieren. Deswe- 
13 Die Firma Sound $\mathrm{GmbH}$ stellt CDs her. Sie möchte ihren Gewinn vergrößern. Daher wurde eine Unternehmensberatung mit einer Marktanalyse beauftragt. Es wurde u. a. festgestellt, dass sich der monatliche Gewinn G der aktuellen CD in Abhängigkeit vom Verkaufspreis p einer $C D$ durch die Funktion $G(p)=-300 p^{2}+6000 p-20000$

modellieren lässt. Wenn G negativ ist, bedeutet das, dass die Firma einen Verlust macht.

a) Zeichne den Graphen der Gewinnfunktion G für $p$ von 0 bis 30 .

b) Welchen Gewinn erzielt die Firma bei einem Stückpreis von $p=14 €(p=8 €)$ ?

c) Zu welchem Preis sollte die Firma das Produkt verkaufen, um einen möglichst großen

Gewinn zu erzielen? Wie groß ist der Gewinn dann?

d) Bei welchem Preis macht die Firma keinen Gewinn, aber auch keinen Verlust (sog.

Break-even-Punkt)?

Abb. 1 Beispielaufgabe kategorisiert als Kostenrechnung (inhaltliche Kategorie) und industriekaufmännischer Kontext (hoher Grad der Berufsbezogenheit) (Neue Wege 9, 2008, S. 11)

Abb. 2 Beispielaufgabe kategorisiert als Kostenrechnung (inhaltliche Kategorie) und andere berufsbezogene kaufmännische Kontexte (mittlerer Grad der Berufsbezogenheit) (Mathe live 8, 2014, S. 39)

\begin{tabular}{l}
\hline $\begin{array}{l}\text { Ein Zeitungsverkäufer hat } 30,00 € \\
\text { Kosten pro Woche. Am Verkauf einer }\end{array}$ \\
Zeitung verdient er $0,05 €$. \\
a) Wie viele Zeitungen muss er mindestens \\
verkaufen, wenn er wöchentlich einen \\
Gewinn von $100,00 €$ erreichen will? \\
b) In einer besonders guten Woche verkauf- \\
te der Zeitungsverkäufer 3000 Zeitungen. \\
Wieviel Gewinn hat er in dieser Woche \\
gemacht?
\end{tabular}

gen fällt diese Lerngelegenheit in die kontextuelle Kategorie andere berufsbezogene kaufmännische Berufe (mittlerer Grad an Berufsbezogenheit).

Die Aufgabe in Abb. 3 behandelt schließlich einen kaufmännisch relevanten Inhaltsbereich, der gemäß der oben dargelegten Kategorisierung im Bereich Finanzierung/Investition liegt. Allerdings wird in der Aufgabe nicht die Perspektive eines kaufmännischen Unternehmens, sondern die einer Privatperson gewählt. Das Signalwort „Sparbuch“ stützt die Einsortierung in die Kontextkategorie private/nicht eindeutige kaufmännische Kontexte (niedriger Grad der Berufsbezogenheit).

Für die leitende Fragestellung in dieser Studie nach berufsspezifischen Lerngelegenheiten im allgemeinbildenden Unterricht sind die Aufgaben mit Zuordnung zu den Kategorien industriekaufmännische Kontexte und andere berufsbezogene kaufmännische Kontexte von Interesse. Sie können eine authentische Lerngelegenheit für berufsfeldbezogene mathematische Kompetenzen nach Neumann et al. (2013) darstellen. Zwar sind diese Aufgaben vom mathematischen Standpunkt aus mit allgemeinbildenden mathematischen Kompetenzen lösbar - sie entstammen ja auch einschlägigen Schulbüchern -, jedoch wird durch die Situierung in kaufmännischen Kontexten auf außerschulische, klar kaufmännisch berufliche Anforderungssituationen verwiesen. Bei Aufgaben der Kategorie private/nicht eindeutige kaufmännische 
10 a) Im Januar möchte Romana sich die Zinsen für das Vorjahr in ihrem Sparbuch eintragen lassen. Ihr Guthaben betrug über das ganze Jahr 372,68 Euro, der Zinssatz $1 \frac{1}{4} \%$.

b) Ihr Bruder hat sich die Zinsen schon am Tag vorher gutschreiben lassen; er schaut in seinem Sparbuch nach und vergleicht: Ich habe 2,91 Euro weniger bekommen als du.

Wie hoch war sein Sparguthaben (bei gleichem Zinssatz)?

Abb. 3 Beispielaufgabe kategorisiert als Finanzierung/Investition (inhaltliche Kategorie) und private/ nicht eindeutige kaufmännische Kontexte (niedriger Grad der Berufsbezogenheit) (Mathe Netz 6 2005, S. 178)

Kontexte wird kein Bezug zu beruflichen Kontexten hergestellt, sodass sie in dieser Studie nicht als berufsspezifische Lerngelegenheiten verstanden werden.

Alle Aufgaben wurden auf diese Weise nach mathematischem Inhaltsbereich und Grad der Berufsbezogenheit kategorisiert. Aus jeder Schulbuchreihe wurde ein Buch zweitkodiert, sodass bei etwa $22 \%$ der Aufgaben eine unabhängige Zweitkodierung vorliegt und entsprechende Interrater-Reliabilitäten sowohl für den Schritt der Aufgabenidentifikation als auch für die Aufgabenklassifikation betrachtet werden können: Im Identifikationsschritt ergab sich eine prozentuale Übereinstimmung von $p_{0}=0,99$ sowie ein Cohens Kappa von $\kappa=0,95$, sodass das Cut-Off-Kriterium von 0,75 erfüllt ist (Fleiss et al. 2003), die Identifikation relevanter Aufgaben also intersubjektiv mit hoher Übereinstimmung gelang. Anschließend wurden die identifizierten Aufgaben nach ihrem mathematischen Inhalt und ihrem Grad der Berufsbezogenheit in den vier bzw. drei Kategorien klassifiziert. Die Interrater-Reliabilität der Zuordnung in das zwölfteilige Kategoriensystem (s. Tab. 2) lag bei $p_{0}=0,82$ und $\kappa=0,76$, sodass Identifikation und Klassifizierung der Aufgaben ebenfalls als objektiv eingeschätzt werden können.

\section{Ergebnisse}

In den 18 Lehrbüchern wurden insgesamt 1154 Aufgaben mit kaufmännischem Kontext (von etwa 20.000) identifiziert. Mit Blick auf Forschungsfrage 1 liegt damit der Anteil der Aufgaben, die überhaupt in einem kaufmännischen Kontext stehen,

Tab. 2 Verteilung der identifizierten Aufgaben bezüglich inhaltlicher Kategorie und Grad der Berufsbezogenheit, kursiv: berufsspezifische Lerngelegenheiten im engeren Sinne

\begin{tabular}{lllll}
\hline Mathematischer Inhalt & \multicolumn{2}{l}{ Anzahl an Aufgaben mit Grad der Berufsbezogenheit } & Gesamt \\
& Hoch & Mittel & Niedrig & \\
\hline Kostenrechnung & 29 & 37 & 371 & 437 \\
Leistungsrechnung & 53 & 35 & 265 & 353 \\
Abschreibung & 0 & 0 & 12 & 12 \\
Finanzierung/ & 49 & 6 & 297 & 352 \\
Investition & & & & \\
Gesamt & 131 & 78 & 945 & 1154 \\
In \% & $11,4 \%$ & $6,8 \%$ & $81,8 \%$ & $100 \%$ \\
\hline
\end{tabular}


bei knapp 6\%. Da 33\% aller Aufgaben generell kontextualisiert sind, decken die Aufgaben im kaufmännischen Kontext hiervon etwa $17 \%$ ab.

Tab. 2 zeigt die Verteilung der 1154 identifizierten Aufgaben auf die 12 Kategorien. Mit Blick auf die zweite Forschungsfrage wird bei $82 \%$ der identifizierten Lerngelegenheiten mit kaufmännischem Kontext nicht die Sicht eines kaufmännischen Unternehmens eingenommen, sondern die Kontexte sind der Kategorie niedrige Berufsbezogenheit (private/nicht eindeutige kaufmännische Kontexte) zuzuordnen. Während der Anteil der spezifisch industriekaufmännischen Kontexte bei $11 \%$ lag (hoher Grad an Berufsbezogenheit), werden andere berufsbezogene kaufmännische Kontexte in ca. 7\% der Aufgaben thematisiert (mittlerer Grad). Gemessen an allen Aufgaben weisen also nur ca. 1\% der Aufgaben mittleren oder hohen Berufsbezug auf und können als Lerngelegenheiten für berufsfeldbezogene mathematische Kompetenzen im engeren Sinne verstanden werden (Tab. 2, kursiv gesetzte Werte). Gemessen an den kontextualisierten Aufgaben sind dies ca. $3 \%$.

Mit Blick auf die inhaltliche Charakterisierung berichten wir hier die Verteilung der Aufgaben auf berufliche Handlungsfelder. Dabei zeigen sich kaum Lerngelegenheiten für die inhaltliche Kategorie Abschreibung, die zudem alle mit niedrigem Grad der Berufsbezogenheit einhergehen. Die restlichen Aufgaben verteilen sich etwa gleichmäßig auf die weiteren drei Inhaltskategorien, wobei die Verteilung in Bezug auf den Grad des Berufsbezugs keine Auffälligkeiten aufweist.

Bezüglich der Unterschiede zwischen den verschiedenen Klassenstufen (dritte Forschungsfrage) zeigt Tab. 3 zunächst deskriptiv eine Abnahme der Aufgaben mit kaufmännischem Kontext bei zunehmender Klassenstufe. In den Stufen 6 und 7 findet sich etwa dreimal so viele Aufgaben wie in den Stufen 9 und 10, wo im Schnitt nur 31 (bzw. 38) solcher Aufgaben pro Buch vorkommen. Die Verteilungen der Lerngelegenheiten auf die drei Kontext-Kategorien unterscheiden sich dabei zwischen den Klassenstufen nicht signifikant $\left(\chi^{2}(d f=8)=13,90, p=0,08\right)$. Es ist also zusammenfassend entgegen der Erwartung nicht zu beobachten, dass in Schulbüchern für höhere Klassenstufen mehr Berufsbezug angeboten wird.

Die verschiedenen Schulbuchreihen unterscheiden sich deutlich (Tab. 4). Die Verteilungen der Kontextkategorien sind nicht homogen $\left(\chi^{2}(d f=6)=73,15, p<0,001\right)$. Während für drei der Schulbuchreihen (Neue Wege, Mathe Netz, mathelive) die Verteilungen des Grads der Berufsbezogenheit von der aggregierten Verteilung der jeweils anderen Schulbuchreihen abweichen $\left(40,19<\chi^{2}(d f=2)<59,22, p<0,001\right)$, repräsentiert die Reihe Lambacher Schweizer eher die insgesamt beobachtete Ver-

Tab. 3 Anzahl der identifizierten Aufgaben nach Klassenstufe und Grad der Berufsbezogenheit

\begin{tabular}{|c|c|c|c|c|}
\hline & \multirow{2}{*}{$\begin{array}{l}\text { Durchschnittliche Anzahl iden- } \\
\text { tifizierter Aufgaben je Buch }\end{array}$} & \multicolumn{3}{|c|}{ Anzahl an Aufgaben mit Grad der Berufsbezogenheit } \\
\hline & & Hoch & Mittel & Niedrig \\
\hline Klasse 6 & 100,5 & $32^{a}$ & $17^{a}$ & $152^{a}$ \\
\hline Klasse 7 & 104,25 & 43 & 26 & 348 \\
\hline Klasse 8 & 64,75 & 26 & 18 & 215 \\
\hline Klasse 9 & 31,25 & 7 & 10 & 108 \\
\hline Klasse 10 & 38 & 23 & 7 & 122 \\
\hline
\end{tabular}

a In Klassenstufe 6 wurden nur zwei statt vier Schulbücher betrachtet 
Tab. 4 Durchschnittliche Anzahl der identifizierten Aufgaben pro Buch und Anteil der Aufgaben nach Grad der Berufsbezogenheit je Schulbuchreihe

\begin{tabular}{lllll}
\hline & $\begin{array}{l}\text { Durchschnittliche } \\
\text { Anzahl identifizierter } \\
\text { Aufgaben je Buch }\end{array}$ & $\begin{array}{l}\text { Anteil an Aufgaben mit Grad der Berufsbezogen- } \\
\text { heit } \\
\text { Hoch }\end{array}$ & Mittel & Niedrig \\
\hline Neue Wege 6-10 & 54,6 & 0,13 & 0,13 & 0,74 \\
Mathe Netz 6-10 & 63 & 0,19 & 0,05 & 0,76 \\
Lambacher Schwei- & 57,5 & 0,12 & 0,07 & 0,81 \\
zer 7-10 & & & & 0,03 \\
mathelive 7-10 & 84 & 0,02 & 0,03 & 0,94 \\
\hline
\end{tabular}

teilung $\left(\chi^{2}(d f=2)=0,08, p=0,96\right)$. Die drei Schulbuchreihen, die primär für die gymnasiale Sekundarstufe I konzipiert wurden (Neue Wege, Mathe Netz, Lambacher Schweizer) weisen dabei mit 19-26\% ähnliche Anteile von Aufgaben mit hoher und mittlerer Berufsbezogenheit auf. In der Reihe Neue Wege werden mehr Aufgaben aus anderen berufsbezogenen kaufmännischen Kontexten angeboten, während Mathe Netz einen besonders hohen Anteil spezifisch industriekaufmännischer Aufgaben beinhaltet. Bei der Reihe mathelive ist zwar die durchschnittliche Anzahl der Aufgaben mit mindestens mittlerem Grad an Berufsbezogenheit je Buch größer (84 zu 55-63 Aufgaben), diese liegen aber mit noch deutlicherem Schwerpunkt in der Kategorie mit niedrigem Berufsbezug (94\% private/nicht eindeutige kaufmännische Kontexte). Es ist also nicht zu beobachten, dass die Schulbuchreihen, die sich primär an gymnasiale Bildungsgänge wenden, weniger berufsfeldbezogene mathematische Lerngelegenheiten aufweisen als die Reihe, die sich auch an Bildungsgänge für den Mittleren Schulabschluss wendet. Es sei aber nochmals drauf hingewiesen, dass die Anzahl der identifizierten Aufgaben, die im Anschluss in Bezug auf den Grad der Berufsbezogenheit kategorisiert wurden, insgesamt gering ist.

\section{Diskussion}

Der literacy-orientierte allgemeinbildende Mathematikunterricht zielt theoretisch auf den Erwerb mathematischer Kompetenzen, die für den Übergang in die berufliche Erstausbildung als relevant angesehen werden. Obwohl der allgemeinbildende Mathematikunterricht also u. a. dem Ziel der Berufsvorbereitung dienen soll, gibt es derzeit wenig Forschungsbefunde zu zugehörigen Lerngelegenheiten. In der hier berichteten Studie wurde unseres Wissens erstmalig dieses Desiderat aufgegriffen und exemplarisch für die mathematiknahen kaufmännischen Berufe bearbeitet. Dazu wurde das Konzept der berufsfeldbezogenen mathematischen Kompetenzen genutzt, die als mathematische Modellierungskompetenzen in beruflichen Kontexten angesehen werden können (vgl. Neumann et al. 2013). Ausgehend von den Ergebnissen der Transferforschung ist zu vermuten, dass die Reichweite von Modellierungskompetenzen von den genutzten Erwerbskontexten abhängt. Aus der Perspektive der Berufsausbildung stellt sich demnach die Frage, inwiefern Kontexte mit Potenzial zur Berufsvorbereitung im Mathematikunterricht der allgemeinbildenden Schule vorkommen. Da Schulbücher als potentiell implementiertes Curriculum angesehen 
werden (vgl. Valverde et al. 2002), diente die Analyse einschlägiger Schulbuchreihen in dieser Studie als Annäherung an den Mathematikunterricht und ermöglichte einen Überblick über die Charakteristik entsprechender Lerngelegenheiten. Dabei wurde beispielhaft der Beruf der Industriekaufleute als Referenzrahmen herangezogen, da er zu den beliebtesten Ausbildungsberufen in Deutschland im kaufmännischen Bereich gehört.

Die Ergebnisse zeigten, dass über alle analysierten Schulbuchreihen und Klassenstufen hinweg ein Anteil von nur 6\% der fast 20.000 Untersuchungseinheiten in einem kaufmännischen Kontext stehen. Knapp ein Fünftel dieser Aufgaben mit kaufmännischem Kontext, also etwa $1 \%$ aller Aufgaben, sind dabei aus der Perspektive eines kaufmännischen Unternehmens dargestellt und können in Anlehnung an das Kompetenzmodell von Neumann et al. (2013) als Lerngelegenheit für berufsfeldbezogene Kompetenzen und damit im engeren Sinne als berufsvorbereitend angesehen werden. Trotz der Anwendungsorientierung im Unterricht (33\% der Aufgaben sind kontextualisiert, $6 \%$ der Aufgaben haben einen kaufmännischen Kontext) weist nur ein verschwindend geringer Anteil also tatsächlich einen Berufsbezug auf. Es ist dabei hervorzuheben, dass kaufmännische Kontexte mit zu den häufigsten Anwendungsbereichen im Mathematikunterricht gehören, da sie sich bei vielen zentralen Inhalten (z. B. Bruchrechnung, Messen, Prozentrechnung, Dreisatz, Funktionen) als Realbezüge anbieten und ein Großteil der Ausbildungsplätze in Deutschland im kaufmännischen Bereich angeboten werden, somit die in der Studie fokussierten Themen also durchweg als relevant eingeschätzt werden können.

Zwischen der frühen (Klassenstufen 6 \& 7) sowie der späten (Klassenstufen 9 \& 10) Sekundarstufe I gibt es erhebliche Unterschiede in der Quantität der Lerngelegenheiten mit kaufmännischem Kontext. Allerdings weisen die Schulbücher entgegen der Erwartung nicht mehr potentiell berufsvorbereitende Lerngelegenheiten in zeitlicher Nähe zum Übergang in die berufliche Ausbildung auf, sondern diese sind sogar eher in den unteren Klassenstufen zu verorten. In einem Mathematikbuch der Klassenstufe 9, also für viele Schülerinnen und Schüler unmittelbar vor dem Übergang in die berufliche Erstausbildung, kommen deswegen durchschnittlich vier Aufgaben mit konkretem kaufmännischem Berufsbezug vor. Eine Erklärung für diese Beobachtungen sind die inhaltlichen Bereiche Prozentrechnung und Dreisatz, die eine wichtige Basis der kaufmännischen Ausbildung darstellen, jedoch im allgemeinbildenden Mathematikunterricht in den Klassenstufen 6 \& 7 unterrichtet werden. Die fachlich-curriculare Strukturierung der Schulbücher dominiert hier gegenüber Zielen der Berufsvorbereitung oder einer Orientierung an den Bedürfnissen der Lernenden verschiedener Altersstufen. Es konnte daneben eine substanzielle Varianz zwischen den Schulbuchreihen beobachtet werden. Obwohl es einen breiteren Diskurs über Realitätsbezüge im Mathematikunterricht gibt, der auch feinkörnig zwischen verschiedenen Aufgabentypen unterscheidet (Leuders und Leiß 2006), ist uns eine Diskussion konkreter Gestaltungsfragen in Bezug auf genutzte Kontexte und die mögliche Umsetzung von Berufsorientierung mit Hilfe von authentischen Anforderungen nicht bekannt und stellt ein Desiderat der mathematikdidaktischen und wirtschaftspädagogischen Forschung dar.

Die kriteriale Bewertung, welcher Anteil berufsbezogener Aufgaben adäquat ist, ist deswegen schwierig und passende Richtgrößen sind kaum verfügbar. Im Sinne 
der Cognitive flexibility theory sollen im Unterricht unterschiedliche Kontexte genutzt werden, die Situated cognition theory legt die Anwendung von Mathematik in authentischen Kontexten nahe. Angesichts der mannigfaltigen potenziellen Bezugspunkte des allgemeinbildenden Mathematikunterrichts besteht die Schwierigkeit darin, ein geeignetes Maß zwischen Aufgabenvielfalt und Orientierung bezüglich wichtiger Berufsfelder zu finden, zumal authentische Anforderungen aufwändiger zu bearbeiten sind als Aufgaben mit einfacher zugänglichen privaten oder wenig berufsspezifischen Kontexten. In PISA werden für den Mathematiktest vier Kontextkategorien, darunter occupational (OECD 2017), genutzt. Ähnlich sind dort für den Bereich der Financial Literacy vier Kategorien individual, home and familiy, societal und education and work rahmengebend (OECD 2017). In den Tests wird jeweils eine Balancierung der Aufgaben über diese Bereiche angestrebt. Entsprechend sollen dort $25 \%$ der Aufgaben aus den Bereichen occupational (Mathematik) bzw. education and work (Financial Literacy) kommen. Für den allgemeinbildenden Unterricht erscheinen $25 \%$ als Kennziffer für die spezifisch beruflichen Lerngelegenheiten zu hoch. Wie zu Beginn des Artikels aufgezeigt, können aber kaufmännische Berufe im Allgemeinen (58\% aller Ausbildungsverträge) wegen der hohen Bedeutsamkeit im Ausbildungssystem in Deutschland als wichtige Referenz für den Mathematikunterricht verstanden werden und beispielsweise ein Orientierungswert von $10 \%$ für den Anteil der berufsbezogenen Aufgaben an den allgemein kaufmännisch kontextuierten Aufgaben angelegt werden. Damit könnten neben diversen anderen beruflichen Lerngelegenheiten auch allgemeinere gesellschaftliche, wissenschaftliche sowie private Lerngelegenheiten mit kaufmännischem Bezug im Mathematikunterricht realisiert werden. Allerdings liegt der in der Studie ermittelte Wert $(6 \%)$ noch deutlich niedriger.

Insgesamt zeigt die Schulbuchanalyse zwar Potential zur Vorbereitung auf kaufmännische Berufe im allgemeinbildenden Unterricht auf, bei genauerer Betrachtung sind die Aufgaben hauptsächlich in deutlich privat konnotierten Situationen kontextualisiert und es ist fraglich, inwiefern dies zur Berufsvorbereitung im engeren Sinne beitragen kann.

Die hier dargelegte Studie verwendet die Anzahl der Aufgaben, die auf berufsfeldbezogene mathematische Kompetenzen abzielen, als Maß für die Vorbereitung auf kaufmännische Ausbildungsberufe. Der bisher rein quantifizierende Zugang kann hier erste Erkenntnisse zur Rolle beruflicher Kontexte liefern, unterliegt jedoch auch deutlichen Einschränkungen. Da in dieser Studie Schulbücher im Sinne des potentiell implementierten Curriculums zur Charakterisierung von Merkmalen des allgemeinbildenden Mathematikunterrichts genutzt wurden, liegen keine Informationen darüber vor, wie Lehrkräfte die Aufgaben tatsächlich im Mathematikunterricht umsetzen. Es ist nicht auszuschließen, dass unterschiedliche Teilbereiche von Schulbüchern im implementierten Curriculum in unterschiedlicher Intensität genutzt werden, sodass unsere Befunde die tatsächlich realisierten beruflichen Lerngelegenheiten über- oder unterschätzen können. Erkenntnisse liegen dazu nicht vor.

Unsere Studie fokussiert zudem ausschließlich kaufmännische berufliche Anforderungen (und beispielhaft industriekaufmännische Anforderungen) und daher können keine Aussagen über potentiell berufsorientierende Lerngelegenheiten für andere Berufsfelder abgeleitet werden. Wenn sich für andere Felder ein ähnlich ho- 
her Grad an Aufgaben aus dem privat/nicht eindeutigen Bereich zeigt, so könnte dies ein Ansatzpunkt zur geforderten Stärkung der Berufsorientierung sein (Matthes und Ulrich 2014).

Einschränkend ist weiter zu erwähnen, dass die Charakterisierung von Lerngelegenheiten als berufsvorbereitend in unserer Studie auf theoretischen Argumenten beruht, die bisher empirisch nicht abgesichert sind. So steht beispielsweise der Nachweis aus, ob Aufgaben, denen in unserer Studie ein hoher Grad an Berufsbezogenheit zugewiesen wurde, für Schülerinnen und Schüler tatsächlich passende Lerngelegenheiten darstellen, wozu beispielsweise auch gehört, ob diese für Lernende interessant sind. Andrerseits wäre zu eruieren, inwiefern die Auseinandersetzung mit Aufgaben mit privat/nicht eindeutigen Kontexten gegebenenfalls trotzdem zur Ausbildung berufsrelevanter Kompetenzen beitragen kann.

Auch aus inhaltlicher Perspektive liefert die vorliegende Studie nur eingeschränkt Informationen. Erkenntnisse über die Qualität der Lerngelegenheiten, beispielsweise inwiefern die in den Kontexten genutzten Situationen zentrale berufliche Anforderungen abdecken oder die Realitätsbezüge authentisch sind, wurden in dem ersten Zugriff noch nicht gewonnen. Der klassifizierte Grad der Berufsbezogenheit schafft hier Anhaltspunkte, jedoch könnten tiefergehende inhaltliche Analysen zusätzliche Erkenntnisse über die Art der berufsbezogenen Lerngelegenheiten im Mathematikunterricht liefern.

Offen bleibt bisher auch, ob das Konstrukt der berufsfeldbezogenen mathematischen Kompetenzen und allgemeiner das Kompetenzmodell von Neumann et al. (2013) tatsächlich geeignet ist, um Fragen der Passung am Übergang Schule Beruf abzubilden. Es bleibt zu prüfen, ob solche berufsfeldbezogenen mathematischen Kompetenzen, sofern sie denn bei (zukünftigen) Auszubildenden schon in der Schule erworben wurden, auch tatsächlich die theoretisch anzunehmende Rolle für einen erfolgreichen Übergang Schule - Beruf spielen. Das Projekt ManKobE (Mathematisch-naturwissenschaftliche Kompetenzen in der beruflichen Erstausbildung) untersucht dazu in einer groß angelegten Längsschnittstudie für verschiedene Ausbildungsberufe (u. a. Industriekaufleute) die Bedingungen sowie die Entwicklung der mathematischen und beruflichen Kompetenzen im Ausbildungsverlauf.

Trotz der Limitationen liefert die hier vorgestellte Studie beispielhaft an kaufmännischen Ausbildungsberufen erste Erkenntnisse zu Art und Umfang der berufsbezogenen Lerngelegenheiten im Mathematikunterricht der allgemeinbildenden Schule. Sie kann so zu einem notwendigen Diskurs über die Ausgestaltung des Ziels Berufsvorbereitung im allgemeinbildenden Mathematikunterricht beitragen.

Funding Open Access funding provided by Projekt DEAL.

Open Access Dieser Artikel wird unter der Creative Commons Namensnennung 4.0 International Lizenz veröffentlicht, welche die Nutzung, Vervielfältigung, Bearbeitung, Verbreitung und Wiedergabe in jeglichem Medium und Format erlaubt, sofern Sie den/die ursprünglichen Autor(en) und die Quelle ordnungsgemäß nennen, einen Link zur Creative Commons Lizenz beifügen und angeben, ob Änderungen vorgenommen wurden.

Die in diesem Artikel enthaltenen Bilder und sonstiges Drittmaterial unterliegen ebenfalls der genannten Creative Commons Lizenz, sofern sich aus der Abbildungslegende nichts anderes ergibt. Sofern das betreffende Material nicht unter der genannten Creative Commons Lizenz steht und die betreffende Handlung nicht nach gesetzlichen Vorschriften erlaubt ist, ist für die oben aufgeführten Weiterverwendungen des Materials die Einwilligung des jeweiligen Rechteinhabers einzuholen. 
Weitere Details zur Lizenz entnehmen Sie bitte der Lizenzinformation auf http://creativecommons.org/ licenses/by/4.0/deed.de.

\section{Literatur}

\section{Verwendete Literatur}

Bundesinstitut für Berufsbildung (BIBB). (2018). Datenreport zum Berufsbildungsbericht 2018. Bonn: BMBF.

Blum, W., \& Leiß, D. (2005). Modellieren im Unterricht mit der „Tanken“-Aufgabe. Mathematik lehren, $128,18-21$.

Bundesministerium für Bildung und Forschung (BMBF). (2002). Verordnung über die Berufsausbildung zum Industriekaufmann/zur Industriekauffrau. Bundesgesetzblatt, 51(1), 2764-2774.

Brown, J.S., Collins, A., \& Duguid, P. (1989). Situated cognition and the culture of learning. Educational researcher, 18(1), 32-42.

Euler, D., \& Hahn, A. (2014). Wirtschaftsdidaktik. Bern: Haupt.

Fleiss, J.L., Levin, B., \& Paik, M.C. (2003). Statistical methods for rates and proportions. New Jersey: John Wiley \& Sons.

van den Ham, A.-K., \& Heinze, A. (2018). Does the textbook matter? Longitudinal effects of textbook choice on primary school students' achievement in mathematics. Studies in Educational Evaluation, $59,133-140$.

Heinze, A., \& Grüßing, M. (Hrsg.). (2009). Mathematiklernen vom Kindergarten bis zum Studium. Kontinuität und Kohärenz als Herausforderung beim Mathematiklernen. Münster: Waxmann.

von Hering, R., Rietenberg, A., Heinze, A., \& Lindmeier, A. (2018). The applied knowledge of trainees as industrial clerks solving problems with vocational and non-vocational context. In E. Bergqvist, M. Österholm, C. Granberg \& L. Sumpter (Hrsg.), Proceedings of the 42nd Conference of the International Group for the Psychology of Mathematics Education (S. 179-186). Umeå: PME.

Heymann, H.W. (1996). Allgemeinbildung und Mathematik. Weinheim: Beltz.

Jongebloed, H.-C. (2011). Der Mensch als Zweck der Bildung - Übergänge zwischen allgemeinbildender und beruflicher Schule - Bestandsaufnahme sowie Probleme und Chancen. Profil-Das Magazin für Gymnasium und Gesellschaft, 18(6), 12-29.

Jordan, A., Ross, N., Krauss, S., Baumert, J., Blum, W., \& Neubrand, M. (2006). Klassifikationsschema für Mathematikaufgaben: Dokumentation der Aufgabenklassifikation im COACTIV-Projekt. Berlin: Max-Planck-Institut für Bildungsforschung.

Klieme, E., \& Leutner, D. (2006). Kompetenzmodelle zur Erfassung individueller Lernergebnisse und zur Bilanzierung von Bildungsprozessen. Zeitschrift für Pädagogik, 52(6), 876-903.

Klieme, E., Neubrand, M., \& Lüdtke, O. (2001). Mathematische Grundbildung: Testkonzeption und Ergebnisse. In PISA-Konsortium (Hrsg.), PISA 2000. Basiskompetenzen von Schülerinnen und Schülern im internationalen Vergleich (S. 139-190). Opladen: Leske + Budrich.

KMK (2002). Rahmenlehrplan für den Ausbildungsberuf Industriekaufmann/Industriekauffrau. Bonn: KMK.

KMK (2003). Bildungsstandards im Fach Mathematik für den Mittleren Schulabschluss. Bonn: KMK.

Lehmann, R., \& Seeber, S. (Hrsg.). (2007). ULME III. Untersuchung von Leistungen, Motivation und Einstellungen der Schülerinnen und Schüler in den Abschlussklassen der Berufsschulen. Hamburg: Behörde für Bildung und Sport.

Leuders, T., \& Leiß, D. (2006). Realitätsbezüge. In W. Blum, C. Drüke-Noe, R. Hartung \& O. Köller (Hrsg.), Bildungsstandards Mathematik: konkret (S. 194-206). Berlin: Cornelsen.

Matthes, S., \& Ulrich, J.G. (2014). Wachsende Passungsprobleme auf dem Arbeitsmarkt. Berufsbildung in Wissenschaft und Praxis, 43(1), 5-7.

Mullis, I., Martin, M., Foy, P., \& Arora, A. (2012). TIMSS 2011 international results in mathematics. Chestnut Hill: Boston College.

Münk, D. (2013). Übergang Schule - Berufsausbildung: Strukturen im Bildungssystem - Übergangsstrukturen mit System? In G. Bellenberg \& M. Forell (Hrsg.), Bildungsübergänge gestalten. Ein Dialog zwischen Wissenschaft und Praxis (S. 251-256). Münster: Waxmann.

Neubrand, J. (2002). Eine Klassifikation mathematischer Aufgaben zur Analyse von Unterrichtssituationen: Selbsttätiges Arbeiten in Schülerphasen in den Stunden der TIMSS-Video-Studie. Hildesheim: Franzbecker. 
Neumann, K., Vollstedt, M., Lindmeier, A., Bernholt, S., Eckhardt, M., \& Harms, U. (2013). Strukturmodelle allgemeiner Kompetenz in Mathematik und den Naturwissenschaften und Implikationen für die Kompetenzentwicklung im Rahmen der beruflichen Ausbildung in ausgewählten kaufmännischen und gewerblich-technischen Berufen. In R. Nickolaus, J. Retelsdorf, E. Winther \& O. Köller (Hrsg.), Mathematisch-naturwissenschaftliche Kompetenzen in der beruflichen Erstausbildung. Stand der Forschung und Desiderata (Zeitschrift für Berufs- und Wirtschaftspädagogik, Bd. 26, S. 113-137). Stuttgart: Steiner.

Nickolaus, R. (2011). Kompetenzmessung und Prüfungen in der beruflichen Bildung. Zeitschrift für Berufs- und Wirtschaftspädagogik, 107(2), 161-173.

Nickolaus, R., \& Seeber, S. (2013). Berufliche Kompetenzen: Modellierungen und diagnostische Verfahren. In A. Frey, U. Lissmann \& B. Schwarz (Hrsg.), Handbuch berufspädagogischer Diagnostik. Weinheim: Beltz.

Niss, M., \& Højgaard, T. (2011). Competencies and Mathematical Learning. Ideas and inspiration for the development of mathematics teaching and learning in Denmark. Roskilde: IMFUFA.

OECD (2003). The PISA 2003 assessment framework: Mathematics, reading, science and problem solving knowledge and skills. Paris: OECD.

OECD (2017). PISA 2015. Technical Report. Paris: OECD.

Perkins, D. N., \& Salomon, G. (1992). Transfer of learning. International Encyclopedia of Education, 2, 6452-6457.

Rezat, S. (2009). Das Mathematikbuch als Instrument des Schülers. Eine Studie zur Schulbuchnutzung in den Sekundarstufen. Wiesbaden: Vieweg + Teubner.

Schmidt, W. H., Curtis, C. M., Houang, R. T., Wang, H. C., Wiley, D. E., \& Cogen, L. S. (2001). Why schools matter: A crossnational comparison of curriculum and learning. San Francisco: Jossey-Bass.

Siebert, U., \& Heinze, A. (2016). Modellierung mathematischer Kompetenzen von Industriekaufleuten am Übergang in die berufliche Erstausbildung. In Institut für Mathematik und Informatik Heidelberg (Hrsg.), Beiträge zum Mathematikunterricht 2016 (S. 1293-1296). Münster: WTM.

Spiro, R. J., Coulson, R.L., Feltovich, P.J., \& Anderson, D. K. (1988). Cognitive flexibility theory: advanced knowledge acquisition in ill-structured domains. Technical Report No. 441

Valverde, G. A., Bianchi, L. J., Wolfe, R. G., Schmidt, W.H., \& Houang, R. T. (2002). According to the book. Using TIMSS to investigate the translation of policy into practice through the world of textbooks. Dordrecht: Kluwer.

Volodina, A., Nagy, G., \& Köller, O. (2015). Success in the first phase of the vocational career: The role of cognitive and scholastic abilities, personality factors, and vocational interests. Journal of Vocational Behavior, 91, 11-22.

Winther, E. (2010). Kompetenzmessung in der beruflichen Bildung. Bielefeld: wbv.

\section{Untersuchte Schulbücher}

Baum, M. et al. (2006). Lambacher Schweizer 7. Mathematik für Gymnasien. Ausgabe A (1. Aufl.). Stuttgart: Klett.

Baum, M. et al. (2007). Lambacher Schweizer 8. Mathematik für Gymnasien. Ausgabe A (1. Aufl.). Stuttgart: Klett.

Baum, M. et al. (2008). Lambacher Schweizer 9. Mathematik für Gymnasien. Ausgabe A (1. Aufl.). Stuttgart: Klett.

Baum, M. et al. (2009). Lambacher Schweizer 10. Mathematik für Gymnasien. Ausgabe A (1. Aufl.). Stuttgart: Klett.

Böer, H. et al. (2008). Mathe live 9. Erweiterungskurs. Mathematik für die Sekundarstufe I (1. Aufl.). Stuttgart: Klett.

Böer, H. et al. (2009). Mathe live 10. Erweiterungskurs. Mathematik für die Sekundarstufe I (1. Aufl.). Stuttgart: Klett.

Böer, H. et al. (2013). Mathe live 7. Mathematik für die Sekundarstufe I. Ausgabe N (1. Aufl.). Stuttgart: Klett.

Böer, H. et al. (2014). Mathe live 8. Mathematik für die Sekundarstufe I. Ausgabe N (1. Aufl.). Stuttgart: Klett.

Cukrowicz, J. et al. (2005). MatheNetz Gymnasium 6. Ausgabe N. Braunschweig: Westermann.

Cukrowicz, J. et al. (2006). MatheNetz Gymnasium 7. Ausgabe N. Braunschweig: Westermann.

Cukrowicz, J. et al. (2007). MatheNetz Gymnasium 8. Ausgabe N. Braunschweig: Westermann. 
Cukrowicz, J. et al. (2008). MatheNetz Gymnasium 9. Ausgabe N. Braunschweig: Westermann.

Cukrowicz, J. et al. (2009). MatheNetz Gymnasium 10. Ausgabe N. Braunschweig: Westermann.

Lergenmüller, A., \& Schmidt, G. (2005). Mathematik Neue Wege 6. Arbeitsbuch für Gymnasien. Niedersachsen. Braunschweig: Schroedel.

Lergenmüller, A., \& Schmidt, G. (2006). Mathematik Neue Wege 7. Arbeitsbuch für Gymnasien. Niedersachsen. Braunschweig: Schroedel.

Lergenmüller, A., \& Schmidt, G. (2007). Mathematik Neue Wege 8. Arbeitsbuch für Gymnasien. Niedersachsen. Braunschweig: Schroedel.

Lergenmüller, A., \& Schmidt, G. (2008). Mathematik Neue Wege 9. Arbeitsbuch für Gymnasien. Niedersachsen. Braunschweig: Schroedel.

Lergenmüller, A., \& Schmidt, G. (2009). Mathematik Neue Wege 10. Arbeitsbuch für Gymnasien. Niedersachsen. Braunschweig: Schroedel. 